\title{
Assistência à saúde de gestantes usuárias de crack: um protocolo de revisão de
}

\section{escopo}

\author{
Health assistance to pregnant crack users: a scope review protocol \\ Asistencia a la salud de gestantes usuarias de crack: un protocolo de revisión de alcance
}

Recebido: 26/01/2022 | Revisado: 03/02/2022 | Aceito: 04/02/2022 | Publicado: 06/02/2022

Heliandra Linhares Aragão

ORCID: https://orcid.org/ 0000-0001-6881-7250

Universidade Federal do Ceará, Brasil

E-mail: heliandrabj@gmail.com

Eliany Nazaré Oliveira

ORCID: https://orcid.org/0000-0002-6408-7243

Universidade Estadual Vale do Acaraú, Brasi E-mail: elianyy@gmail.com

Maria Adelane Monteiro da Silva

ORCID: https://orcid.org/0000-0001-7579-2645

Universidade Estadual Vale do Acaraú, Brasil

E-mail: adelanemonteiro@hotmail.com

Joyce Mazza Nunes Aragão

ORCID: https://orcid.org/0000-0003-2865-579X

Universidade Estadual Vale do Acaraú, Brasi

E-mail: joycemazza5@gmail.com

Lídia Cristina Monteiro da Silva

ORCID: https://orcid.org/ 0000-0003-0403-6839

Universidade Estadual Vale do Acaraú, Brasil

E-mail: lidia2021o@outlook.com

Maria Alanne de Oliveira Frota

ORCID: https://orcid.org/ 0000-0002-7993-6658

Centro de Atendimento Psicossocial Álcool e Outras Drogas de Sobral, Brasil

E-mail: alannefrotapsi@gmail.com

Douglas Prado Araújo

ORCID: http://orcid.org/ 0000-0002-6195-521X

Centro de Atendimento Psicossocial Álcool e Outras Drogas de Sobral, Brasil

E-mail: douglaspradomsn@ hotmail.com

Leila Ponte Vasconcelos

ORCID: http://orcid.org/ 0000-0002-9069-6309

Centro de Atendimento Psicossocial Álcool e Outras Drogas de Sobral, Brasil

E-mail: leilapontepsi@gmail.com

Júlio César de Oliveira Santos

ORCID: https://orcid.org/ 0000-0001-6097-9597

Centro de Atendimento Psicossocial Álcool e Outras Drogas de Sobral, Brasil

E-mail: enfjuliosantos@gmail.com

Edijane Araújo da Silva

ORCID: https://orcid.org/ 0000-0001-5647-7658

Centro de Atendimento Psicossocial Álcool e Outras Drogas de Sobral, Brasil

E-mail: edijane120588@gmail.com

\begin{abstract}
Resumo
A gestação se caracteriza como um processo natural, fisiológico e único, vivenciado pela mulher, ocasionando transformações de cunho biopsicossocial, caracterizando-se por anseios e expectativas pela chegada do bebê, mudanças físicas e alterações psicológicas. O consumo do álcool, durante o período gestacional, ocasiona riscos clínicos preocupantes para o binômio mãe-feto. Para a mãe, o álcool funciona como inibidor de apetite, sendo relevante acompanhamento de pré-natal efetivo, pois devido a essa condição, a gestante pode obter prejuízos na ingestão de uma alimentação necessária, ocorrendo a não captação dos nutrientes e favorecendo o surgimento de carência nutricional ao feto. O objetivo desse protocolo é apresentar etapas para a construção de uma revisão de escopo, com intuito de mapear as evidências sobre a assistência à saúde às gestantes usuárias de crack. Esse protocolo tem como base as recomendações do Manual do Instituto Joana Briggs. Serão utilizadas as bases de dados: PUBMED, LILACS, SCIELO, BDEnf, Web of Science e Scopus e a Biblioteca Digital de Teses e Dissertações da CAPES para as buscas de dados. As informações serão coletadas na fase de extração sendo armazenadas em banco de dados eletrônico, criado no programa criado no programa Microsoft Excel for Windows ${ }^{\circledR}$ versão 2019. A criação do referido
\end{abstract}


protocolo possibilitará a realização de uma revisão de escopo, metodologicamente embasada nos princípios estabelecidos pelo manual do Instituto Joanna Briggs e, a partir dela, a obtenção de um maior conhecimento acerca da assistência à saúde de gestantes usuárias de crack

Palavras chaves: Gestantes de risco; Cocaína crack; Assistência à saúde; Atenção à saúde; Protocolo de pesquisa.

\begin{abstract}
Pregnancy is characterized as a natural, physiological and unique process experienced by women, causing biopsychosocial transformations, characterized by yearnings and expectations for the baby arrival, physical changes, and psychological changes. Alcohol consumption during the gestational period causes unsettling clinical risks for the maternal-fetal dyad. For the mother, alcohol works as an appetite suppressant, which makes an effective prenatal follow-up even more relevant, since due to this condition, a pregnant woman can suffer losses in the necessary food intake, causing non-capturing of nutrients and favoring the fetus nutritional deficiency. This protocol objective is to present steps for a scope review construction aiming to map evidences on health care for pregnant crack users. This protocol is based on Joana Briggs Institute Manual recommendations. The following databases will be used for data search: PUBMED, LILACS, SCIELO, BDEnf, Web of Science and Scopus, and CAPES Digital Library of Theses and Dissertations. Information will be collected in the extraction phase and then stored in an electronic database, created in Microsoft Excel for Windows ${ }^{\circledR}$ version 2019. This protocol inception will enable scope review methodologically based on principles established by Joanna Briggs Institute Manual and, starting from there, greater knowledge about health care for pregnant crack users.
\end{abstract}

Keywords: Pregnancy, High-risk; Crack cocaine; Delivery of health care; Protocol.

\title{
Resumen
}

La gestación se caracteriza por ser un proceso natural, fisiológico y único, vivenciado por la mujer, ocasionando transformaciones de tipo biopsicosocial, caracterizándose por deseos y expectativas por la llegada do bebé, cambios físicos y alteraciones psicológicas. El consumo de alcohol, durante el período gestacional, ocasiona riesgos clínicos preocupantes para el binomio madre-feto. Para la madre, el alcohol funciona como inhibidor del apetito, siendo relevante un seguimiento efectivo del prenatal, pues debido a esta condición, la gestante puede perjudicarse en la ingestión de una alimentación necesaria, ocurriendo la no captación de los nutrientes y favoreciendo el surgimiento de carencia nutricional en el feto. El objetivo de este protocolo es presentar etapas para la construcción de una revisión de alcance, con el propósito de mapear las evidencias sobre la asistencia a la salud de las gestantes usuarias de crack. Este protocolo tiene como base las recomendaciones del Manual del Instituto Joana Briggs. Serán utilizadas las bases de datos: PUBMED, LILACS, SCIELO, BDEnf, Web of Science y Scopus y la Biblioteca Digital de Tesis y Disertaciones de la CAPES para las buscas de datos. Las informaciones serán recolectadas en la fase de extracción siendo almacenadas en banco de datos electrónico, criado en el programa microsoft excel for windows® versión 2019. La creación del referido protocolo posibilitará la realización de una revisión de alcance, metodológicamente basada en los principios establecidos por el Manual del Instituto Joanna Briggs y, a partir de ahí, la obtención de un mayor conocimiento sobre la asistencia a la salud de gestantes usuarias de crack

Palabras clave: Gestantes de riesgo; Cocaína crack; Asistencia a la salud; Atención a la salud; Protocolo de investigación.

\section{Introdução}

A gestação se caracteriza como um processo natural, fisiológico e único, vivenciado pela mulher, ocasionando transformações de cunho biopsicossocial, caracterizando-se por anseios e expectativas pela chegada do bebê, mudanças físicas e alterações psicológicas (Lopes et al., 2019). Embora seja considerado um processo fisiológico, algumas gestações podem implicar evolução desfavorável. Podendo desencadear alterações na morfologia e no sofrimento fetal, assim, frisa-se o uso de Substâncias Psicoativas (SPA), sendo contraindicadas no período gestacional, podendo ocasionar danos permanentes à gestante e ao feto (Silva et al., 2020).

O consumo do álcool, durante o período gestacional, ocasiona riscos clínicos preocupantes para o binômio mãe-feto. Para a mãe, o álcool funciona como inibidor de apetite, sendo relevante acompanhamento de pré-natal efetivo, pois devido a essa condição, a gestante pode obter prejuízos na ingestão de uma alimentação necessária, ocorrendo a não captação dos nutrientes e favorecendo o surgimento de carência nutricional. No feto, a substância atravessa a barreira placentária, ocasionando risco de hidrocefalia, má formação fetal, alterações no sistema nervoso do feto, como também doenças e deficiências permanentes (Capelletti et al., 2019). Já o tabaco não apresentou riscos direcionados à mãe, porém pode desencadear aborto espontâneo, baixo peso ao nascer, problemas respiratórios e hipóxia fetal. No que se refere à maconha, 
identificou-se aumento do apetite, perda da inibição, diminuição da memória e euforia ou sensação de relaxamento nas mães, assim como afetou o desenvolvimento do tubo neural do feto e anencefalias. A cocaína, por sua vez, pode ocasionar diminuição do fluxo sanguíneo da mãe para o útero, placenta e feto, desencadeando aborto espontâneo, parto prematuro, crescimento intrauterino retardado e sofrimento fetal grave. Por fim, o crack pode causar alterações graves no sistema nervoso central da mãe e do feto.

Mastroianni et al. (2019) elucidam que o uso de SPA em gestantes está relacionado a dificuldades e impasses vivenciados nesse período, considerando a droga uma "valva de escape" que possibilita lidar com as adversidades e angústias. Assim, a permanência do uso, durante a gestação, pode desencadear negações ou sentimentos ambivalentes em relação à maternidade, enquanto a abstinência permite identificar a influência do uso no exercício dos cuidados maternos. Camargo et al. (2019) apontam que o contexto cultural, o histórico familiar, a relação com o companheiro, o planejamento da gestação e as redes de apoio, também, possuem influência nesse processo.

Dessarte, o cenário atual identifica comumente mulheres que vivenciam o período gestacional como usuárias dependentes de substâncias psicoativas, apresentando histórico de consumo prévio ao evento da gravidez, colaborando para enfrentamento considerado como grande obstáculo, quando se trata de evitar o uso durante a gestação (Rocha \& Rocha, 2019). Assim, o uso da substância psicoativa, no decorrer da gestação, tem corroborado o surgimento de complicações para o binômio mãe-feto, tendo em vista o alcance da velocidade em que a droga atinge a corrente sanguínea. Desta forma, as gestantes usuárias de crack apresentam classificação elevada de risco, favorecendo, assim, possíveis eventualidades, com intercorrências obstétricas (Wronski et al., 2016).

As gestantes usuárias de crack, geralmente, têm baixa adesão ao pré-natal, o que aumenta os riscos de intercorrências maternas e fetais. A gestação nestas circunstâncias pode ser responsável pelo baixo peso do recém-nascido, além de causar aumento no número de partos prematuros (Silva et al., 2020). O consumo de cocaína/crack, ao longo da gestação, tem contribuído significativamente para o desencadeamento de riscos de complicações à saúde materna. No entanto, esses riscos ultrapassam os agravos obstétricos, como também colaboram com danos que atingem consideravelmente à saúde neonatal, infantil e adulta desses conceptos. Enfatiza-se, portanto, o comportamento de gestantes usuárias de drogas, pois, geralmente, iniciam tardiamente o pré-natal, o que desencadeia um processo de prejuízos, como menor acompanhamento da gestação, favorecendo intercorrências clínicas (Ferreira \& Miranda, 2016).

Assim, é de extrema importância a utilização de estratégias, como a busca ativa das pacientes usuárias faltosas em consultas de pré-natal, bem como a execução do acompanhamento de equipes multidisciplinares, de modo a construir adesão concreta de gestantes usuárias nos tratamentos indicados, contribuindo para efetivação de assistência integral positiva (Antunes et al., 2018).

A assistência prestada às gestantes que usam álcool e/ou outras drogas é considerada de alta complexidade, exigindo formação específica dos profissionais de saúde que prestarão esse cuidado. Cada gestante apresenta características singulares, deste modo, os profissionais devem dispor de habilidades, como estar atentos e capacitados para saber atuar com as demandas psicológicas e sociais destas mulheres (Maia et al., 2019; Camargo et al., 2018).

Maia et al. (2019) destacam em estudo que é fundamental que essas gestantes tenham acesso a uma rede de atenção à saúde que atendam às suas necessidades. De acordo com a Organização Pan-Americana da Saúde (2011), as redes de atenção à saúde produzem resultados positivos, como redução da fragmentação da atenção; melhoria da eficiência global do sistema; resposta melhor às necessidades e às expectativas das pessoas; melhora do custo efetividade dos serviços de saúde; redução das hospitalizações desnecessárias; diminuição da utilização excessiva de serviços e exames; atenuação do tempo de permanência hospitalar; aumento da satisfação dos usuários e do autocuidado. 
As deficiências assistenciais para gestantes usuárias de crack instigam o sistema de saúde a formular estratégias de acolhimento, além de adotar dispositivos de aproximação, possibilitando a eficiente atuação dos profissionais de saúde, a fim de reduzir as taxas de mortalidade materna e infantil e/ou reduzir os danos gerados pelo uso abusivo de tal substância na gestação.

Assim, ao identificar lacunas de conhecimento na literatura existente, a revisão de escopo torna o processo de divulgação mais explícito, produzindo passo adiante para obtenção de conclusões sobre os estudos produzidos, considerando especialmente o estado das atividades de investigação.

Nos levantamentos realizados até o momento, não se identificou revisão de escopo sobre a assistência à saúde de gestantes usuárias de crack. Desta forma, o estudo proposto torna-se relevante, pois visa identificar alternativa de produção de conhecimento da realidade. Infere-se, ainda, que o estudo possibilitará novas descobertas rumo à efetivação da melhor assistência à população em estudo.

Pretende-se com este projeto delinear a assistência à saúde às gestantes usuárias de crack, buscando descrever as potencialidades e fragilidades. Espera-se que os resultados sirvam de base para qualificar o atendimento ao público referido, compreendendo a relevância do problema do uso do crack em Sobral-CE, com vistas à redução da mortalidade materna e infantil, além da prevenção dos possíveis danos aos bebês acarretados pelo uso do crack na gestação.

Para isso, optou-se metodologicamente pela revisão de literatura, com ênfase em abordagem específica denominada revisão de escopo. A proposta desta dissertação se organiza na apresentação da contextualização do que se tem produzido sobre assistência à saúde de gestantes usuárias de crack.

\subsection{Pergunta da pesquisa}

Para construção da pergunta de pesquisa, utilizou-se da estratégia Population, Concept e Context (PCC) para uma scoping review (Aromataris \& Munn, 2020). Definiram-se: P- Gestante usuárias de crack; C- Gestantes de risco; e CAssistência à saúde. Com base nessas definições, estabeleceu-se a pergunta norteadora: qual a produção científica sobre assistência à saúde de gestantes usuárias de crack? A fim de identificar as potencialidades e os limites, o arcabouço teórico e as pesquisas configuraram-se o objeto de estudo da pesquisa de mestrado proposta.

Quadro 1. Estratégia PCC.

\begin{tabular}{|c|c|c|c|}
\hline Problema & \multicolumn{3}{|c|}{ Qual a produção científica sobre assistência à saúde de gestantes usuárias de crack? } \\
\hline & População & Conceito & Contexto \\
\hline Conceitos & $\begin{array}{l}\text { Gestantes usuárias de } \\
\text { crack }\end{array}$ & Gestantes de risco & Assistência à Saúde \\
\hline Tradução & $\begin{array}{l}\text { Crack-using pregnant } \\
\text { women }\end{array}$ & Risk pregnant & Delivery of Health Care \\
\hline Combinação & $\begin{array}{ll}\text { "Pregnant"; } & \text { "Pregnant } \\
\text { Crack-using" } & \\
\end{array}$ & $\begin{array}{l}\text { "Risk pregnant"; "Pregnancy } \\
\text { High-Risk" }\end{array}$ & $\begin{array}{l}\text { "Delivery of Health Care"; } \\
\text { "Health"; "Care" }\end{array}$ \\
\hline Construção & $\begin{array}{l}\text { "Pregnant" OR "Pregnant } \\
\text { Crack-using" }\end{array}$ & $\begin{array}{lcc}\text { "Risk pregnant" } & \text { AND } \\
\text { "Pregnancy High-Risk" } & \end{array}$ & $\begin{array}{l}\text { "Delivery of Health Care" AND } \\
\text { "Health" AND "Care" }\end{array}$ \\
\hline $\begin{array}{l}\text { Uso da estratégia com } \\
\text { os booleanos }\end{array}$ & $\begin{array}{l}\text { ("Pregnant" OR "Pregnant } \\
\text { AND ("Delivery of Health }\end{array}$ & $\begin{array}{l}\text { Crack-using") AND ("Risk pregn } \\
\text { Care" AND "Health" AND "Care" }\end{array}$ & ant" AND "Pregnancy High-Risk") \\
\hline
\end{tabular}

Fonte: Autores. 
O Quadro 1 trás o delineamento da estratégia PCC proposta, mostrando os conceitos, tradução, combinação e construção, além de apresentar a estratégia unida com os operadores booleanos. Facilitando, assim, a visualização do que iremos buscar.

\subsection{Critérios de inclusão}

O ponto de partida é identificar a questão da pesquisa a ser respondida, como um caminho norteador que fornece o roteiro para as etapas subsequentes. Os aspectos relevantes da questão devem ser claramente definidos, pois eles têm ramificações para estratégias de pesquisa. As questões de pesquisa são de natureza ampla, pois procuram fornecer maior amplitude de cobertura (Arksey \& O'malley, 2005).

\subsubsection{População}

A POPULAÇÃO de interesse serão gestantes usuárias de crack, atendidas no sistema público de saúde do Brasil. A gestação é um período de grandes mudanças físicas e psicológicas para a mulher. Durante esta fase, ela passa por vivências que irão influenciar intensamente a vida, envolvendo estrutura de personalidade, história pessoal, capacidade de resolução de conflitos, circunstâncias em que ocorre a gestação, características de sua evolução, fator socioeconômico, contexto assistencial, suporte conjugal e familiar e expectativas acerca do bebê (Wronski et al., 2016).

Quando a gestante é usuária de substâncias psicoativas, esse período precisa de maior atenção dos profissionais, pois a dependência química é fator de risco para problemas perinatais, comprometendo a saúde da gestante e o desenvolvimento fetal (Maia et al., 2019).

Para Wronski et al. (2016), no caso de mulheres usuárias de crack, acrescido a esses aspectos, o uso da droga no período gestacional acarreta complicações tanto para a mãe quanto para o feto, devido à rapidez com que essa substância psicoativa chega à corrente sanguínea, ao efeito breve e à dependência. Neste contexto, as gestantes usuárias de crack são consideradas de alto risco, pois têm mais chances de apresentar intercorrências, demandando assistência integral e humanizada por parte da equipe envolvida com o tratamento e a reabilitação.

\subsubsection{Conceito}

O CONCEITO de gestante de risco utilizado para esta revisão está vinculado ao uso de substâncias psicoativas por este grupo, pois consiste em um dos fatores geradores de risco gestacional para a mãe e o feto. Algumas das consequências negativas advindas do uso de drogas ilícitas são a desnutrição e a maior suscetibilidade a infecções, danos que podem se estender ao feto em desenvolvimento (Brasil, 2016).

Para Maia et al. (2019), as mulheres que usam crack são vulneráveis ao HIV e a outras infecções sexualmente transmissíveis, devido ao comportamento sexual de risco, como troca de sexo por substâncias psicoativas, carência do uso de preservativos com múltiplos parceiros, história de vitimização física e sexual, problemas com a justiça criminal, falta de habitação estável e desemprego.

As intervenções na assistência a esse público se baseiam no risco das vulnerabilidades, nos desafios e recursos que quando aumentados, impactam diretamente na saúde.

\subsubsection{Contexto}

O CONTEXTO nesta revisão é a assistência à saúde. O Sistema Único de Saúde tem se deparado com o aumento do número de usuários de substâncias psicoativas, sendo relevante a garantia de continuidade da assistência. 
Existe movimento em busca da construção do sistema de saúde com foco nas Redes de Atenção à Saúde (RAS), em detrimento de sistemas fragmentados. Entende-se as RAS como "arranjos organizativos de ações e serviços de saúde, de diferentes densidades tecnológicas, que integradas por meio de sistemas de apoio técnico, logístico e de gestão, buscam garantir a integralidade do cuidado" (Brasil, 2010).

É necessária a descentralização do cuidado de gestantes usuárias para além da APS, com trabalho compartilhado com os serviços da Rede de Atenção Psicossocial (RAPS), podendo envolver outros pontos da RAS e serviços da rede socioassistencial, conforme demandem os casos, visando assistência de forma integrada (Costa et al., 2015).

O conhecimento acerca de como é percebida a influência do crack na gestação por mulheres usuárias poderá contribuir para um repensar da prática assistencial, qualificando a assistência à saúde.

\subsubsection{Tipo de pontos de evidências}

O método de revisão de escopo proposto permitirá a consulta de várias fontes científicas, portanto, serão considerados estudos observacionais analíticos, incluindo ensaios clínicos, estudos de coorte, transversais, caso - controle e observacionais. Além disso, serão incluídas fontes adicionais provenientes da literatura cinzenta.

\section{Metodologia}

Tratar-se-á de estudo de scoping review, em português, revisão de escopo, conforme o método proposto pelo Instituto Jonna Briggs (JBI) que permite mapear os principais conceitos, clarificar áreas de pesquisa e identificar lacunas do conhecimento, seguindo etapas metodológicas (Aromataris \& Munn, 2020), que seguirá o protocolo registrado no Open Science Framework, conforme o identificador 10.17605/OSF.IO/BV3GM: DOI

Uma revisão de escopo pode ser usada para mapear os principais conceitos subjacentes a um campo de pesquisa, bem como para esclarecer definições de trabalho e/ou os conceitos limites de um tópico, resumir evidências e informar pesquisas futuras (Tricco et al., 2018; Peters et al., 2018).

Revisões de escopo são vantajosas quando se faz necessário sintetizar evidências de pesquisa, além de utilizadas com o fim de mapear a literatura existente em determinado contexto. Além disso, também podem ser utilizadas para resumir e disseminar resultados de pesquisas. As revisões de escopo possuem abordagem ampla, com objetivo de mapear literatura, permitindo, assim, questões de pesquisas mais vastas. O objetivo, por sua vez, das revisões de escopo é mapear os conceitoschave, em determinada área de pesquisa (Aromataris \& Munn, 2020).

\subsection{Estratégias de busca}

Esta etapa envolve a identificação de estudos e o desenvolvimento de um plano de decisão de onde procurar (escolha da base de dados), quais termos usarem (escolha de descritores), quais fontes devem ser pesquisadas, tempo e linguagem. Compreensibilidade e amplitude são importantes na pesquisa. As fontes incluem bases de dados eletrônicas, lista de referências paralelas, busca manual de revistas-chave, organizações e conferências. É importante destacar os aspectos práticos da pesquisa: o tempo, o orçamento e os recursos pessoais mobilizados, já que esses são potenciais fatores limitantes. As decisões precisam ser tomadas antecipadamente e serem explicitadas de modo que fique expresso como os caminhos eleitos afetarão a busca e os resultados (Arksey \& O'malley, 2005, tradução nossa).

Nesse sentido, o levantamento bibliográfico será realizado no período de janeiro a março de 2022. Para adequação às demais bases de dados e plataformas, utilizar-se-ão dos Descritores em Ciências da Saúde (DeCs) para as bases em português: Gestantes de risco; Cocaína crack; Assistência à saúde; Atenção à Saúde, Revisão de escopo. Para as bases de dados que utilizam o idioma inglês, os descritores serão: Pregnancy, High-Risk; Delivery of Health Care; Crack Cocaine. 
Inicialmente, serão analisadas as palavras contidas nos títulos, resumos e descritores. Com realização de busca em bases de dados eletrônicas, lista de referências paralelas e redes existentes, organizações e conferências relevantes. Os estudos selecionados devem responder à questão norteadora desta revisão e serão lidos na íntegra, sendo as referências analisadas, em busca de estudos adicionais.

Junto aos descritores, serão empregados os termos booleanos: AND, OR e NOT, conforme orienta o JBI (Aromataris \& Munn, 2020). A estratégia de busca de dados será elaborada por meio da combinação dos descritores e do uso dos booleanos. Serão incluídos estudos nos idiomas inglês, espanhol e português; com abordagem quantitativa, qualitativa e quanti-qualitativa; estudos primários; revisões sistemáticas, metanálises e/ou metassínteses; e livros, publicados ou disponibilizados de 2015 a 2021. O recorte deste período é visando identificar estudos recentes sobre a temática.

Botelho e Oliveira (2015) realizaram pesquisa que revisou conceitualmente as expressões literatura científica e literatura cinza, além de descreverem algumas das respectivas características. Nos resultados da pesquisa, os autores encontraram que a literatura científica se refere a documentos convencionais ou formais que apresentam facilidades para identificação, divulgação e obtenção, produzidos dentro dos circuitos comerciais.

No mesmo estudo, Botelho e Oliveira (2015) relataram que a literatura cinza são as publicações não comerciais, não convencionais, difíceis de serem encontradas em canais tradicionais de distribuição e que costumam demandar mais pesquisa para localização e recuperação.

\subsection{Fonte de informações}

A seleção dos artigos acontecerá em três etapas: inicialmente, será realizada a leitura de título e resumo, considerando incluir estudos que respondam ao objetivo da pesquisa; na sequência, os estudos selecionados serão lidos na íntegra e, adicionalmente, as referências destes serão avaliadas, considerando captar e incluir estudos que não foram recuperados no momento da busca nas bases de dados selecionadas; na terceira e última etapa, será procedida à leitura completa dos artigos incluídos, tendo em vista destacar e sumarizar as informações que respondam ao objetivo da revisão (Arksey \& O'malley, 2005).

A identificação dos estudos será realizada através das seguintes bases de dado: PUBMED; LILACS; SciELO; BDEnf, Web of Science e SCOPUS. Já a busca por materiais não publicados em periódicos indexados e revisados por pares será realizada na Biblioteca Digital de Teses e dissertações da CAPES. A estratégia de busca conterá os mesmos descritores ou equivalentes e será adaptada para atender a peculiaridades de cada base de dados

\subsection{Seleção dos estudos}

A sistematização das fases da busca, identificação e seleção está representada por meio do Fluxograma Prisma (Figura 1). 
Figura 1 - Modelo de Fluxograma Prisma a ser utilizado no estudo.
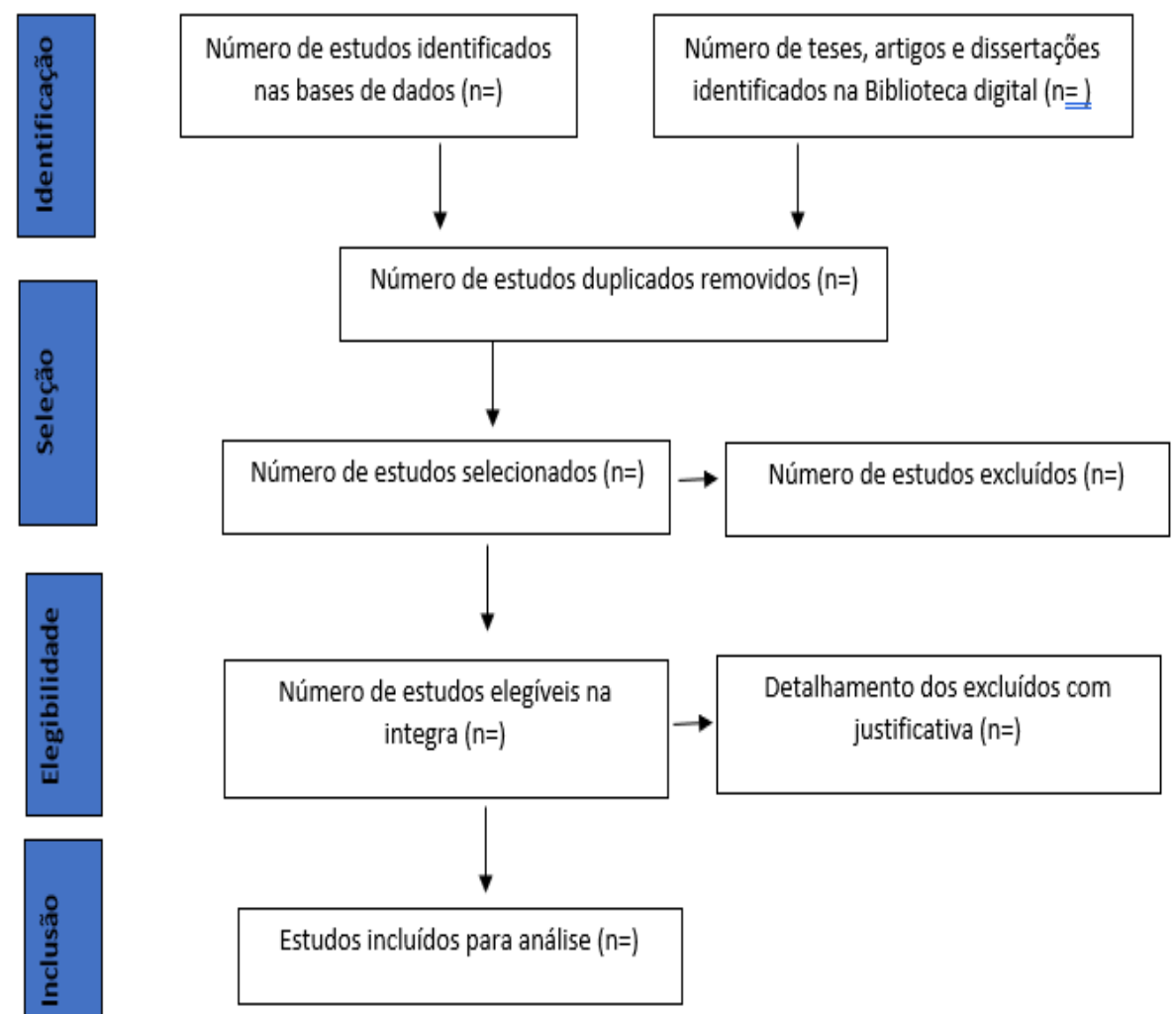

Fonte: Aromataris e Munn (2020).

\section{Resultados e Discussão}

\subsection{Extração dos dados}

Para análise e posterior síntese dos artigos, será utilizado formulário sobre extração de dados, que constarão informações bibliográficas básicas, características dos estudos e informações que permitam compreender como é a assistência à saúde de gestantes usuárias de crack. O formulário de extração será preenchido pelos revisores, de forma independente, as questões e dúvidas sobre as extrações serão tratadas por consenso entre os revisores e, quando não resolvidas, serão discutidas com terceiro revisor (orientador) (Arksey \& O'malley, 2005).

Ressalta-se que o formulário de extração pré-planejado para a revisão proposta será testado e validado, de maneira independente, por dois integrantes da pesquisa, em etapa piloto que incluirá $10 \%$ dos artigos da lista final de estudos recuperados na etapa de seleção. Se necessário, serão realizados ajustes nas categorias/itens de extração, após discussão e consultas aos membros da equipe. Esse procedimento tem por finalidade garantir a consistência da extração de informações (Tricco et al., 2018).

As informações coletadas na fase de extração serão armazenadas em banco de dados eletrônico, criado no programa Microsoft Excel for Windows® versão 2019. Para as buscas, contar-se-á com apoio de três bolsistas, os quais, junto com a autora deste projeto, serão divididos em duas duplas. Salienta-se que as buscas serão as cegas e comparativas, após a conclusão. 


\subsection{Apresentação dos dados}

A sumarização e apresentação dos resultados devem ser realizadas de modo a apresentar efetivamente o panorama das informações recuperadas, a extensão e a natureza da literatura sobre o tema revisado. Na revisão de escopo, os resultados serão apresentados em forma de quadro sintético e formato descritivo, conforme orientações do protocolo PRISMA - extensão para revisão de escopo (Tricco et al., 2018).

O quadro sintético conterá resumo dos estudos revisados, de acordo com as informações contidas/resgatadas no formulário de extração de dados, caracterizando os estudos e apresentando os fatores que dificultam ou potencializam a assistência a gestantes usuárias de crack, no contexto do SUS.

Além disso, será produzida síntese narrativa dos achados relacionados às recomendações e limitações dos estudos, considerando produzir informações úteis para pesquisas futuras sobre o tema. Os dados finais serão apresentados descritivamente e por meio de quadros e gráficos que facilitem a visualização dos resultados.

\section{Conclusão}

A criação do referido protocolo possibilitará a realização de uma revisão de escopo, metodologicamente embasada nos princípios estabelecidos pelo manual do Instituto Joanna Briggs e, a partir dela, a obtenção de um maior conhecimento acerca da assistência à saúde de gestantes usuárias de crack. As informações compiladas neste estudo subsidiarão diversos autores que visam desenvolver estudo tendo como objeto gestantes usuárias de crack. Além disso, espera-se que esta revisão contribua para os surgimentos de outros estudos com metodologia diferentes para melhor compreensão da temática.

Para trabalhos futuros, sugere-se a elaboração de outros protocolos de revisão, com métodos e objetivos e bem definidos, que possibilitem uma visão ampla sobre a temática. Protocolo já registrado na Open Science Framework, conforme o identificador 10.17605/OSF.IO/BV3GM: DOI.

\section{Referências}

Antunes, M. B., Demitto, M. de O., Padovani, C., Elias, K. C. de M., Miranda, A. C. M. de, \& Pelloso, S. M. (2018). Desfecho perinatal em gestantes usuárias de drogas atendidas em um centro especializado. Revista Eletrônica Saúde Mental Álcool e Drogas, 14(4), 211-218. https://doi.org/10.11606/issn.18066976.smad.2018.00037

Aromataris, E., \& Munn, Z. (2020). JBI Manual for Evidence Synthesis. Joanna Briggs Institute. https://doi.org/10.46658/JBIMES-20-01.

Botelho, R. G., \& de Oliveira, C. da C. (2017). Literaturas branca e cinzenta: uma revisão conceitual. Ciência da Informação, 44(3). https://doi.org/10.18225/ci.inf.v44i3.1804

Brasil (2010). Ministério da Saúde. Portaria $n^{\circ}$ 4.279/GM/MS, de 30 de dezembro de 2010, do Ministério da Saúde: Estabelece diretrizes para a organização da Rede de Atenção à Saúde no âmbito do SUS. Ministério da Saúde. https://bvsms.saude.gov.br/bvs/saudelegis/gm/2010/prt4279_30_12_2010.html

Brasil (2016). Ministério da Saúde. Sistema para detecção do uso abusivo e dependência de substâncias psicoativas: Encaminhamento, intervenção breve, reinserção social e acompanhamento. Ministério da Saúde. https://www.supera.org.br/wp-content/uploads/2016/06/SUP9_Guia.pdf

Capeletti, A. de O., Lins, J. P., \& Giotto, A. C. (2019). As intervenções dos profissionais de enfermagem frente a gestantes usuárias de drogas ilícitas e lícitas. Revista de Iniciação Científica e Extensão, 2(Esp.2), 323-328. https://revistasfacesa.senaaires.com.br/index.php/iniciacao-cientifica/article/view/282

Costa, P. H., Colugnati, F. A., \& Ronzani, T. M. (2015). Mental health services assessment in Brazil: systematic literature review. Ciência \& Saúde Coletiva, 20(10), 3243-3253. https://doi.org/10.1590/1413-812320152010.14612014

Lopes, R. S., Lucchese, R., Silva, G. C., Vera, I., Souza, L. M. M., \& Mendonça, R. S. (2019). O período gestacional e transtornos mentais: evidências epidemiológicas. Humanidades \& Tecnologia, 19(1), 35-54. http://revistas.icesp.br/index.php/FINOM_Humanidade_Tecnologia/article/view/932

Maia, J. A., Rodrigues, A. L., Souza, D. R., \& Figueiredo, M. B. (2019). Uso de drogas por mulheres durante o período gestacional. Revista Enfermagem Contemporânea, 8(1), 25-32. https://doi.org/10.17267/2317-3378rec.v8i1.1744

Mastroianni, F. de C., Balsaneli, E., \& Palamin, J. N. (2019). A influência do uso de substâncias psicoativas nos cuidados maternos segundo mães usuárias: um estudo qualitativo. Cadernos Brasileiros de Saúde Mental, 11(28), 151-169. https://periodicos.ufsc.br/index.php/cbsm/article/view/69389

Organização Pan-Americana de Saúde (2011). A atenção à saúde coordenada pela APS, Construindo as redes de atenção no SUS: Contribuições para o debate. Organização Pan-Americana da Saúde. https://iris.paho.org/bitstream/handle/10665.2/18457/9788579670657_por.pdf?sequence=1\&isAllowed=y 
Research, Society and Development, v. 11, n. 2, e59911226296, 2022

(CC BY 4.0) | ISSN 2525-3409 | DOI: http://dx.doi.org/10.33448/rsd-v11i2.26296

Peters, M. D. J., Godfrey, C., McInerney, P., Munn, Z., Tricco, A. C., \& Khalil, H. Chapter 11: Scoping Reviews (2020 version). In E. Aromataris \& Z. Munn (Eds.), JBI Manual for Evidence Synthesis. Joanna Briggs Institute https://doi.org/10.46658/JBIMES-20-12

Rocha, E. T., \& Rocha, R. R. (2019). Drogas na gravidez e consequências em recém-nascidos. Journal of Specialist, 1(2), 1-29. http://www.journalofspecialist.com.br/jos/index.php/jos/article/view/81

Silva, F. T. R., Tamais, M. L. B., Costa, A. B., Melo, S. C. C. S., \& Fernandes, C. A. M. (2020). Prevalence and factors associated with the use of drugs of abuse by pregnant women. Revista Brasileira de Saúde Materno Infantil, 20(4), 1101-1107. https://doi.org/10.1590/1806-93042020000400010

Tricco, A. C., Lillie, E., Zarin, W., O’Brien, K. K., Colquhoun, H., Levac, D., Moher, D., Peters, M. D. J., Horsley, T., Weeks, L., Hempel, S., Akl, E. A., Chang, C., McGowan, J., Stewart, L., Hartling, L., Aldcroft, A., Wilson, M. G., Garritty, C., ... Straus, S. E. (2018). PRISMA extension for scoping reviews (PRISMA-ScR): Checklist and explanation. Annals of Internal Medicine, 169(7), 467-473. https://doi.org/10.7326/M180850/ASSET/IMAGES/LARGE/M180850FF1_FIGURE_METHODS_FLOW_CHART.JPEG

Wronski, J., Pavelski, T., Guimarães, A. N., Zanotelli, S. S., Schneider, J., \& Bonilha, A. L. (2016). Uso do crack na gestação: vivências de mulheres usuárias. Revista de Enfermagem UFPE on line, 10(4), 1231-1239. https://doi.org/10.5205/1981-8963-v10i4a11108p1231-1239-2016 